\title{
Repeated Gene Transfection Impairs the Engraftment of Transplanted Porcine Neonatal Pancreatic Cells
}

\author{
Min Koo Seo ${ }^{1}$, Cheng-Lin Sun², Ji-Won Kim², Kun-Ho Yoon², Suk Kyeong Lee \\ ${ }^{1}$ Research Institute of Immunobiology, Department of Biomedical Sciences, ${ }^{2}$ Division of Endocrinology \& Metabolism, Department of Internal Medicine, The \\ Catholic University of Korea School of Medicine, Seoul, Korea
}

\begin{abstract}
Background: Previously, we reported that neonatal porcine pancreatic cells transfected with hepatocyte growth factor (HGF) gene in an Epstein-Barr virus (EBV)-based plasmid (pEBVHGF) showed improved proliferation and differentiation compared to those of the control. In this study, we examined if pancreatic cells transfected repeatedly with pEBVHGF can be successfully grafted to control blood glucose in a diabetes mouse model.

Methods: Neonatal porcine pancreatic cells were cultured as a monolayer and were transfected with pEBVHGF every other day for a total of three transfections. The transfected pancreatic cells were re-aggregated and transplanted into kidney capsules of diabetic nude mice or normal nude mice. Blood glucose level and body weight were measured every other day after transplantation. The engraftment of the transplanted cells and differentiation into beta cells were assessed using immunohistochemistry.

Results: Re-aggregation of the pancreatic cells before transplantation improved engraftment of the cells and facilitated neovascularization of the graft. Right before transplantation, pancreatic cells that were transfected with pEBVHGF and then re-aggregated showed ductal cell marker expression. However, ductal cells disappeared and the cells underwent fibrosis in a diabetes mouse model two to five weeks after transplantation; these mice also did not show controlled blood glucose levels. Furthermore, pancreatic cells transplanted into nude mice with normal blood glucose showed poor graft survival regardless of the type of transfected plasmid (pCEP4, pHGF, or pEBVHGF).

Conclusion: For clinical application of transfected neonatal porcine pancreatic cells, further studies are required to develop methods of overcoming the damage for the cells caused by repeated transfection and to re-aggregate them into islet-like structures.
\end{abstract}

Keywords: Diabetes mellitus; Hepatocyte growth factor; Porcine neonatal pancreatic cells; Transfection

\section{INTRODUCTION}

The applications of porcine pancreas and pancreatic cells in the treatment of diabetes have been studied [1-4]. Specifically, there is increasing interest in using neonatal porcine pancreatic cell clusters (NPCCs) due to numerous advantages [5-11]. It was shown that $70 \%$ of the NPCCs of a three-day-old pig were pancreatic duct cells which are known as pancreatic stem cells, and $10 \%$ were beta cells [12]. Glucose was controlled normally in diabetic animal models when NPCCs were transplanted in the clustered state to the mouse kidney capsules [10]. There- fore, NPCCs seem to be a useful source for the future treatment of diabetes. However, it takes four to ten weeks after transplantation for NPCCs to differentiate into beta cells $[10,13]$. Thus, insulin treatment should be continued even after transplantation until hyperglycemia adjusts to normal levels [14]. In addition, the decreasing survival rate of the transplanted NPCCs with long-term exposure to a hyperglycemic environment still remains to be addressed [15].

The hepatocyte growth factor (HGF) was first discovered as a major factor of liver growth [16] and has subsequently been shown to induce the growth of various cells $[17,18]$. HGF is
Corresponding author: Suk Kyeong Lee

Research Institute of Immunobiology, Department of Biomedical Sciences,

The Catholic University of Korea School of Medicine, 505 Banpo-dong,

Seocho-gu, Seoul 137-701, Korea

E-mail: sukklee@catholic.ac.kr

Received: Jul. 28, 2010; Accepted: Oct. 13, 2010
This is an Open Access article distributed under the terms of the Creative Commons Attribution Non-Commercial License (http://creativecommons.org/licenses/by-nc/3.0/) which permits unrestricted non-commercial use, distribution, and reproduction in any medium, provided the original work is properly cited.

Copyright $\odot 2011$ Korean Diabetes Association

http://e-dmj.org 
thought to differentiate NPCCs into beta cells as NPCCs cultured with recombinant HGF showed increased insulin secretion [19].

Monolayer culture of pancreatic cells can facilitate growth factor treatment and gene transfer to promote ductal cell growth and differentiation into beta cells. Single cells separated from NPCCs were cultured in vitro to a certain extent and gene transduced using a retrovirus [12]. The monolayer cultured and re-aggregated pancreatic cells were successfully engrafted in nude mice and part of the cells was confirmed to have differentiated into beta cells four weeks after transplantation [12]. In order to aid the differentiation of NPCCs in vivo, gene transfection and re-aggregation of monolayer cultured pancreatic cells can be performed before transplantation. However, reports of such attempts are rare.

In our previous study, HGF gene was cloned in an EpsteinBarr virus (EBV)-based plasmid (pEBVHGF) and transfected to monolayer cultured pancreatic cells [20]. HGF was expressed about seven times longer in those cells than in the cells transfeced with a conventional plasmid (pHGF). Phosphorylation of the HGF receptor, c-MET, was observed three days after the transfection of pEBVHGF [20]. When pEBVHGF was transfected to pancreatic cells, an increase in insulin secretion was also seen compared with the conventional plasmid transfected cells [20]. EBV-based plasmid contains EBNA-1 and oriP sequences derived from EBV, and these two were confirmed to help the plasmid replicate and remain in the transfected pancreatic cells during cell proliferation [21]. NPCCs are primary cells and transfection efficiency is relatively low. In a preliminary experiment, increased transfection efficiency was achieved when GFP-expressing plasmid (pEBVGFP) was repeatedly transfected to pancreatic cells up to three to four times (data not shown).

In this study, HGF gene cloned in an EBV-based plasmid was repeatedly transfected to pancreatic cells. The cells were then re-aggregated and transplanted into normal nude mice or diabetic nude mice to examine engraftment and differentiation in vivo. Additionally, blood glucose changes of the mice were also analyzed.

\section{METHODS}

\section{Pancreatic cell preparation}

We used Rompun ${ }^{\circledR}(0.15 \mathrm{~mL} / \mathrm{kg}$; Bayer Korea, Seoul, Korea) and Ketamine (16 mL/kg; Yuhan, Seoul, Korea) to anesthetize one- to three-day-old neonatal pigs and removed pancreases. The pancreases were minced using $20 \mathrm{~cm}$ scissors, and digested with collagenase $\mathrm{P}(1 \mathrm{mg} / \mathrm{mL}$ in $\mathrm{HBSS})$ at $37^{\circ} \mathrm{C}$ for 14 minutes. The digested pancreases were filtered using a $500 \mu \mathrm{m}$ mesh, and pancreatic cells that were filtered through the mesh were collected. The pancreas tissue that was not fully digested was placed in $50 \mathrm{~mL}$ tubes and digested again for nine minutes at $37^{\circ} \mathrm{C}$ with collagenase $\mathrm{P}(1 \mathrm{mg} / \mathrm{mL}$ in $\mathrm{HBSS})$. The re-digested tissue was then filtered using the $500 \mu \mathrm{m}$ mesh to collect cells. The isolated pancreatic cells were cultured for one day in a 150 mm culture container with F10 culture medium. The cultured NPCCs were then collected in $50 \mathrm{~mL}$ conical tubes and centrifuged at $37^{\circ} \mathrm{C}$ for nine minutes to remove supernatant prior to adding $10 \mathrm{~mL}$ of cell dissociation solution (Sigma-Aldrich, St. Louis, MO, USA). NPCCs were separated into single cells, and $3 \times 10^{6}$ cells were placed in $100 \mathrm{~mm}$ culture dishes with DMEM (low-glucose) medium. The pancreatic cells were then cultured in an incubator at $37^{\circ} \mathrm{C}$ with $5 \% \mathrm{CO}_{2}$.

\section{Plasmid transfection to monolayer cultured pancreatic cells}

EcoRV and Stu I were used in order to remove the oriP and EBNA-1 sequences from pEBVHGF, resulting in pHGF [20]. An empty EBV-based plasmid, pCEP4, was also used as a control [20]. Plasmid $(5 \mu \mathrm{g})$ and lipofectamine 2000 (Invitrogen, Carlsbad, CA, USA) were mixed each with $500 \mu \mathrm{L}$ Opti-MEM (GIBCO, Grand Island, NY, USA) at room temperature; the two liquids were then carefully mixed together and incubated for 30 minutes.

After separation from neonatal pigs, pancreatic cells were cultured as a monolayer for five days prior to transfection. For transfection, the culture medium was replaced with $5 \mathrm{~mL}$ of serum-free DMEM (low-glucose) and the cells were then incubated with the prepared DNA-lipofectamine 2000 for five hours at $37^{\circ} \mathrm{C}$ and $5 \% \mathrm{CO}_{2}$. The culture medium was changed to DMEM (low-glucose) containing 10\% FBS after five hours, and incubation was continued. Using this method, plasmids were transfected into pancreatic cells three times every two days.

\section{Re-aggregation of the monolayer cultured pancreatic cells} After removing the culture medium, $5 \mathrm{~mL}$ of cell dissociation solution (Sigma-Aldrich) was added to the culture dish and NPCCs were harvested. The cells were placed in a $15 \mathrm{~mL}$ conical tube and centrifuged for 3 minutes at 1,200 rpm. The cells 
grown in eight to ten $100 \mathrm{~mm}$ culture dishes were combined in one $50 \mathrm{~mL}$ conical tube. The cells in the $50 \mathrm{~mL}$ tube was centrifuged for three minutes, the supernatant was removed, and the pancreatic cells were resuspended by adding $1.5 \mathrm{~mL}$ of DMEM (low-glucose). The cells were transferred to a $15 \mathrm{~mL}$ conical tube and were then set with the cover slightly open to carry out re-aggregation for two hours in a $37^{\circ} \mathrm{C}$ incubator with $5 \% \mathrm{CO}_{2}$. The re-aggregated pancreatic cells were then carefully placed in the middle of a $100 \mathrm{~mm}$ culture dish to prevent scattering and incubated for 48 hours before used for transplantation.

\section{Preparation of the diabetes model mice}

The breeding, management, and all of the transplantations of the immunodeficient seven- to eight-week-old mice (CAnN. Cg-Foxn1 nu/Crlj0ri; Orient Bio Inc., Seongnam, Korea) were performed in a Semi-SPF sector. These mice were fasted for eight hours, and $220 \mathrm{mg} / \mathrm{kg}$ of streptozotocin was injected into each animal. Blood from the tail vein was collected every day and blood glucose levels were measured. When the blood glucose level was greater than $400 \mathrm{mg} / \mathrm{dL}$ for five days, the mouse was classified as a diabetic and was used for pancreatic cell transplantation. This research was performed after approval from the Animal Experimentation Ethics Committee of The Catholic University of Korea. Proper procedure and regulations were strictly followed throughout the experimental process.

\section{Transplanting re-aggregated pancreatic cells into mice}

The re-aggregated pancreatic cells were centrifuged for three minutes in $50 \mathrm{~mL}$ conical tubes at 1,200 rpm and the supernatant was removed. The cells were transferred to $1.5 \mathrm{~mL}$ EP-tubes (4,000-5,000 IEq [islet equivalent]) and centrifuged for another three minutes. To transplant the prepared cells, they were let settle at the bottom of PE-50 tube (Becton Dickinson, Franklin Lakes, NJ, USA). Normally, the amount of pancreatic cells required to transplant into one mouse was obtained from one neonatal pig after in vitro culture, plasmid transfection, and re-aggregation processes.

The mice were anesthetized with a peritoneal injection of $0.1 \mathrm{~mL}$ Ketamine and Rompun ${ }^{\circledR}$ mixed in a 5:1 ratio. The kidney was exposed from the mouse through the left flank, and the membrane of the kidney was incised with an injection needle. The pancreatic cells were injected into the membrane of the kidney from the PE-50 tube by applying weak and uni- form pressure using a Hamilton syringe. A high temperature cautery (Bovie Medical Co., St. Petersburg, FL, USA) was used to close the incision. After repositioning the kidney within the body, we sutured the peritoneum and then the skin using punch stitches. After transplantation, we measured weight and blood glucose levels from tail blood draws at two-day intervals, between 4 PM and 5 PM.

\section{Immunohistochemical staining}

The kidneys of pancreatic cell-transplanted mice were fixed with formalin at room temperature for 16 hours and embedded in paraffin. Then, the kidney was sliced into $4 \mu \mathrm{m}$ pieces and mounted onto slides. Paraffin was removed using xylene, and either guinea pig anti-insulin (1:100; Invitrogen) or rabbit anti-pancytokeratin (1:100; Zymed, San Francisco, CA, USA) antibodies were added and incubated at $4^{\circ} \mathrm{C}$ for 16 hours. After that, the slides were reacted at room temperature for two hours with either rhodamine-conjugated anti-guinea pig IgG (1:100; Jackson ImmunoResearch, West Grove, PA, USA) or FITC-conjugated anti-rabbit IgG (1:100; Jackson ImmunoResearch) secondary antibodies. The tissue was covered using mounting solution, which includes DAPI. The tissue was then observed under a fluorescence or confocal microscope.

\section{Cell viability assay}

CCK-8 solution (Cell Counting Kit-8; Dojindo, Kumamoto, Japan) was diluted with fresh culture medium at a 1:10 ratio. The diluted CCK-8 solution was added at $110 \mu \mathrm{L} /$ well to a $96-$ well plate of cultured pancreatic cells and incubated for three hours at $37^{\circ} \mathrm{C}$ with $5 \% \mathrm{CO}_{2}$. The change in the absorbance at $450 \mathrm{~nm}$ was assessed using an ELISA analyzer (Model $680 \mathrm{Mi}-$ croplate Reader; Bio-Rad, Hercules, CA, USA).

\section{RESULTS}

\section{Successful transplantation of the NPCCs which were not manipulated in vitro}

NPCCs isolated as clusters without incubation in vitro or separation into single cells, were transplanted into the kidney capsules of normal nude mice (Fig. 1). From two weeks after transplantation, some of the cells expressed insulin and undifferentiated pancreatic cells were also present. After eight weeks, most of the cells had differentiated into beta cells which formed islets and produced insulin (Fig. 1). 


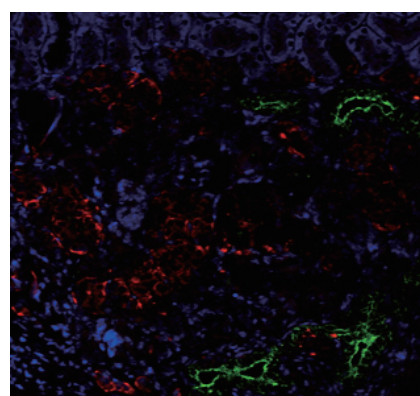

2 week

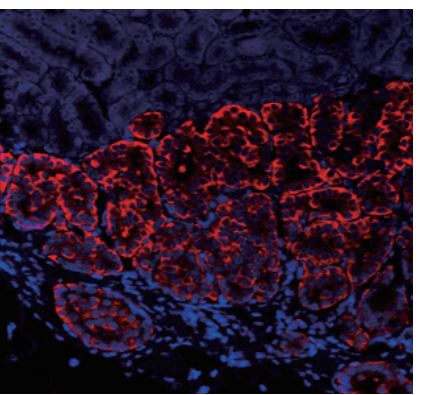

8 week

Fig. 1. Successful transplantation of neonatal porcine pancreatic cell clusters (NPCCs) in normal mice. NPCCs were harvested and transplanted immediately into the kidneys of normal nude mice without any manipulation. Two and eight weeks after transplantation, transplanted NPCCs were examined using immunohistochemistry. Insulin is stained red and pancytokeratin is stained green, while nuclear staining is shown in blue.

\section{Effect of the status of transplanted pancreatic cells on the transplantation efficacy}

After monolayer culture, transplantation efficacy was compared between single cell state and re-aggregated pancreatic cells. Within three days of the birth of the newborn pig, NPCCs were separated and dispersed as single cells. After seven days of monolayer culture, two-day re-aggregation was performed with some cells, and the remaining cells were cultured as a monolayer for two more days. The monolayer cultured pancreatic cells which were harvested as single cells $\left(3.6 \times 10^{6}\right.$ cell/ mouse) or the re-aggregated cells (4,000-5,000 IEq/mouse) were then transplanted into the mouse kidney capsules. The grafts were harvested fourteen days after the transplantation and examined through hymatoxylin staining. The survival rate of transplanted single cells was low in mouse kidney capsules, whereas the re-aggregated pancreatic cells had a high survival rate (Fig. 2, upper panel). Some re-aggregated pancreatic cells differentiated into insulin-secreting beta cells, and blood vessels were generated between transplanted cells (Fig. 2, lower panel).

\section{Effect of repeated transfection on pancreatic cell viability}

Pancreatic cells in monolayer culture were repeatedly transfected with pCEP4, pHGF, or pEBVHGF, and the changes in cell viability were examined. When pancreatic cells were transfected once with plasmid, the cell viability decreased slightly compared to that of the untransfected cells. When the cells were transfected twice, the cell viability decreased further (Fig. 3).

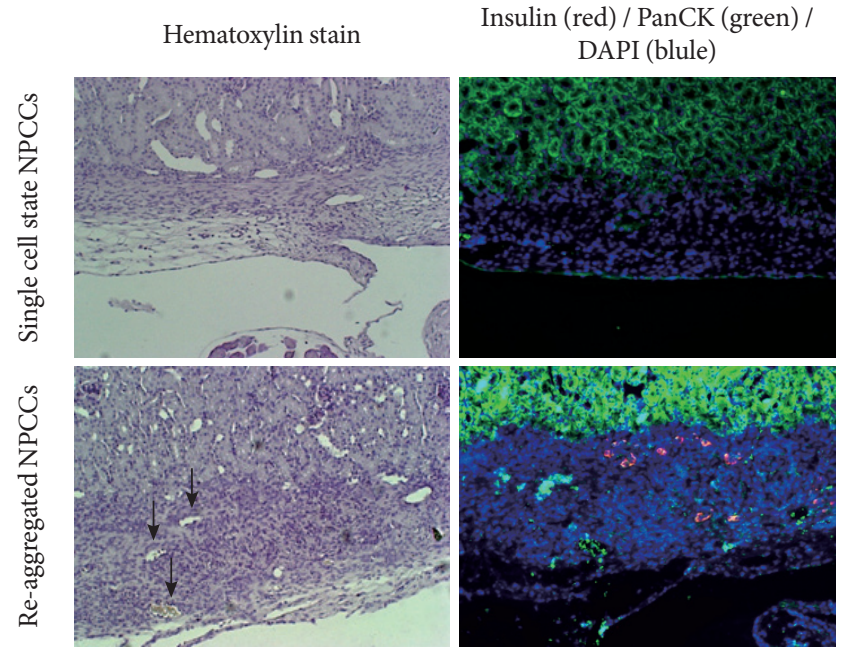

Fig. 2. Effect of re-aggregation on in vivo survival of pancreatic cells after transplantation. Single-cell state or re-aggregated pancreatic cells were transplanted in the kidney capsule of normal mice. The graft was examined following hematoxylin staining or immunohistochemistry two weeks after transplantation. The black arrow point to blood vessels within the graft. Insulin is shown in red and pancytokeratin is shown in green, while nuclear staining is shown in blue. NPCCs, neonatal porcine pancreatic cell clusters.

However, cell viability did not decrease further when transfection was performed three times (Fig. 3). Thus, pancreatic cells were transfected three times for our future experiments.

\section{Changes in blood glucose of diabetic model mice which were transplanted with gene transfected pancreatic cells} Whether the pEBVHGF-transfected pancreatic cells have effect on the treatment of diabetes was examined. For this, body weight and blood glucose changes of the diabetic mice models were measured in two-day intervals after re-aggregated cells which were transfected with pHGF or pEBVHGF were transplanted (4,000 IEq/mouse). We observed that the mice transplanted with cells transfected with any of the plasmids did not recover to their normal weight. However, pEBVHGF group had a tendency to maintain a slightly higher body weight at the early stage when compared to pHGF group (Fig. 4).

\section{Characteristics of the pancreatic cells right after re- aggregation}

The features of the pancreatic cells were analyzed just before transplantation. After consecutive triple pEBVHGF transfection, the cells were re-aggregated for two days. The re-aggre- 

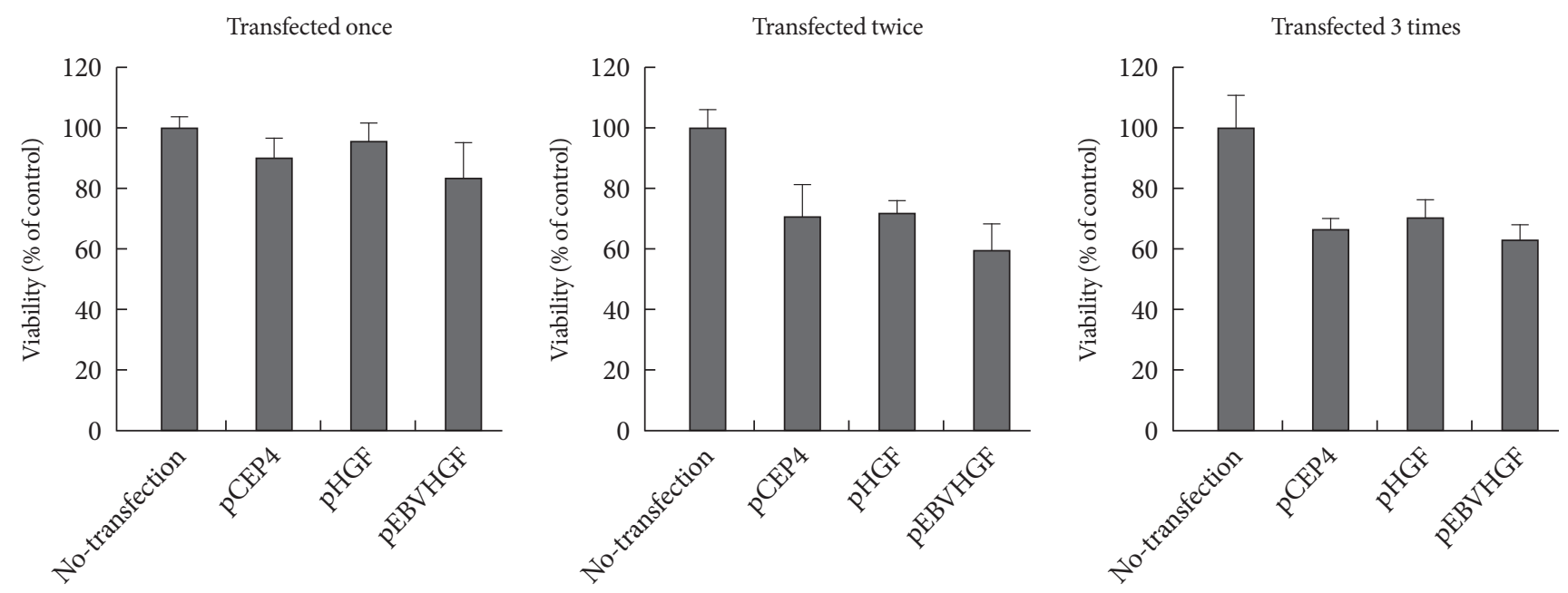

Fig. 3. Effect of repeated transfection on cell viability. Pancreatic cells were transfected repeatedly with pCEP4, pHGF, or pEBVHGF every other day up to three times. Twenty-four hours after each transfection, viable cell count was analyzed using a CCK8 kit. Cell viabilities are expressed as percent of untransfected control cells. Data are presented as the mean \pm standard error of three independent experiments.
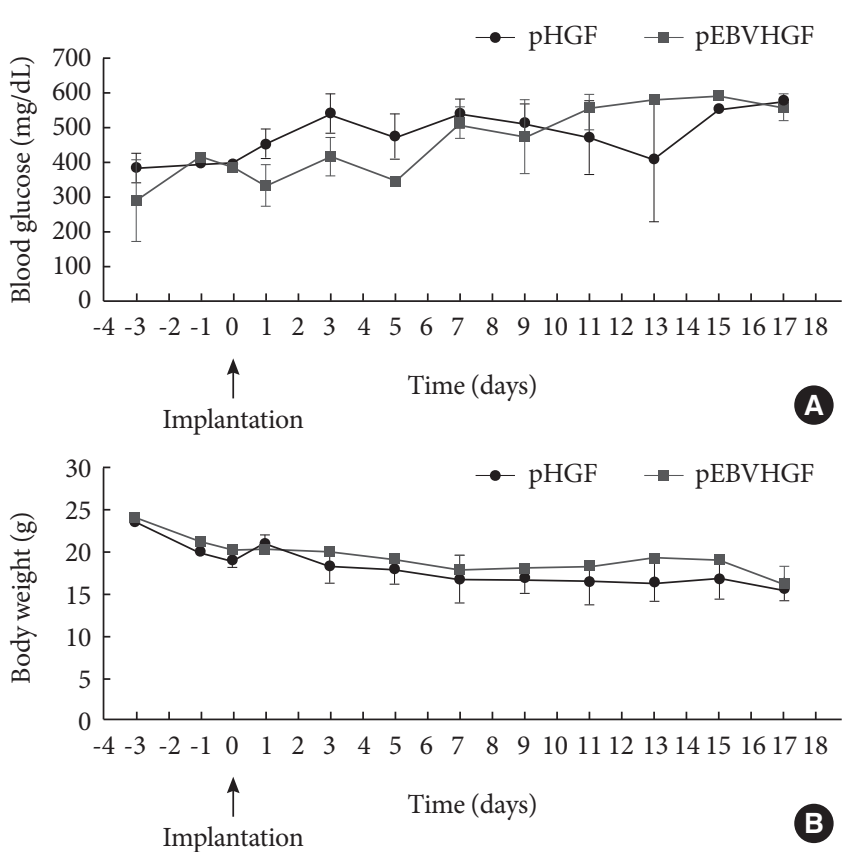

Fig. 4. Concentrations of blood glucose and body weight following transplantation of pancreatic cells transfected with pHGF or pEBVHGF. Pancreatic cells were transfected with pHGF or pEBVHGF, and re-aggregated. The re-aggregated cells were transplanted into the type 1 diabetes mouse model. Blood glucose level (A) and body weight (B) were measured every other day. Time 0 indicates the day of pancreatic cell transplantation.

gated pancreatic cells showed compact round shape, and the expression of the pancreatic cell marker, pancytokeratin, was

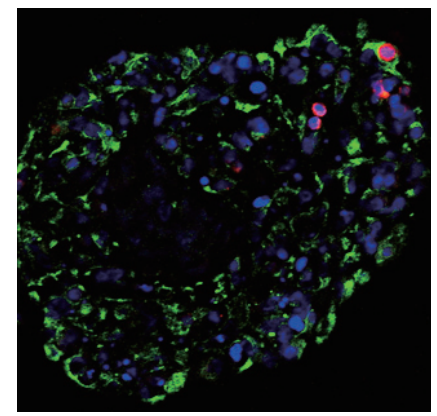

Fig. 5. Pancytokeratin and insulin expressions in the pancreatic cells transfected three times with pEBVHGF and re-aggregated. The cells were fixed and stained right after transplantation into the diabetes mouse model. Insulin is shown in red and pancytokeratin is shown in green, while nuclear staining is shown in blue. Imaging was performed with a confocal microscope ( $400 \times$ objective).

confirmed in most of the cells (Fig. 5). In addition, some insulin-expressing cells were observed (Fig. 5).

\section{Characteristics of the graft after transplantation of pancreatic cells}

After pancreatic cells were transplanted into diabetic mouse model, some mice died of polyuria, hypothermia, and other problems that developed as a result of hyperglycemia during the short observation period. Two out of four mice from the pHGF group and three out of four mice from the pEBVHGF group survived to the completion of the study. The survivors 

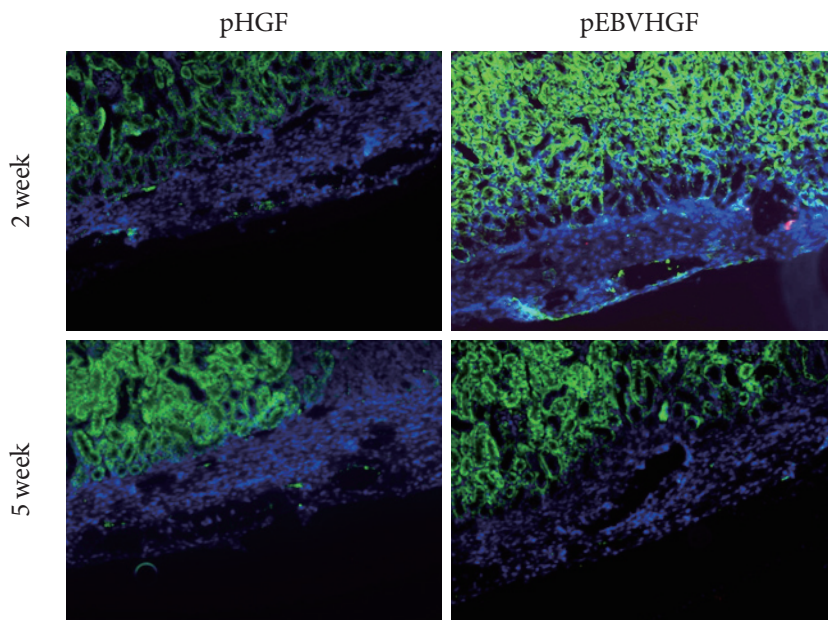

Fig. 6. No expression of insulin or pancytokeratin in the transplanted pancreatic cells in a diabetes mouse model. Pancreatic cells were transfected three times with pHGF or pEBVHGF, re-aggregated, and transplanted into a type 1 diabetes mice. Survival and differentiation of the transplanted cells were examined using immunohistochemistry two and five weeks after transplantation. Insulin is stained red and pancytokeratin is stained green, while nuclear staining is shown in blue.

had their kidneys extracted, and the expressions of insulin and pancytokeratin were confirmed using immunohistochemical staining. Unlike what we observed in Fig. 5, nearly all mouse grafts that survived for two to five weeks underwent fibrosis, and the tissue grafts showed scattered tiny holes (Fig. 6).

\section{Analysis of pancreatic cells transplanted into normal nude mice}

To check whether the low survival rates of pancreatic cells in the diabetic mouse model was due to high glucose toxicity, the cells transfected with pHGF or pEBVHGF were transplanted into normal nude mice. pCEP4 (control plasmid), pHGF, and pEBVHGF were each transfected to pancreatic cells through three transfections each, and then the cells were re-aggregated. The prepared cells were transplanted into normal nude mice (pCEP4 group [ $\mathrm{n}=4]$, pHGF group $[\mathrm{n}=5]$, and pEBVHGF group $[n=5])$. After one, two, and eight weeks, the graft survival was evaluated using immunohistochemical staining. Even in this case, regardless of the type of transfected plasmids, the majority of transplanted cells underwent fibrosis as early as one week after transplantation, leaving only a few insulin-producing cells and pancytokertin positive cells (Fig. 7).

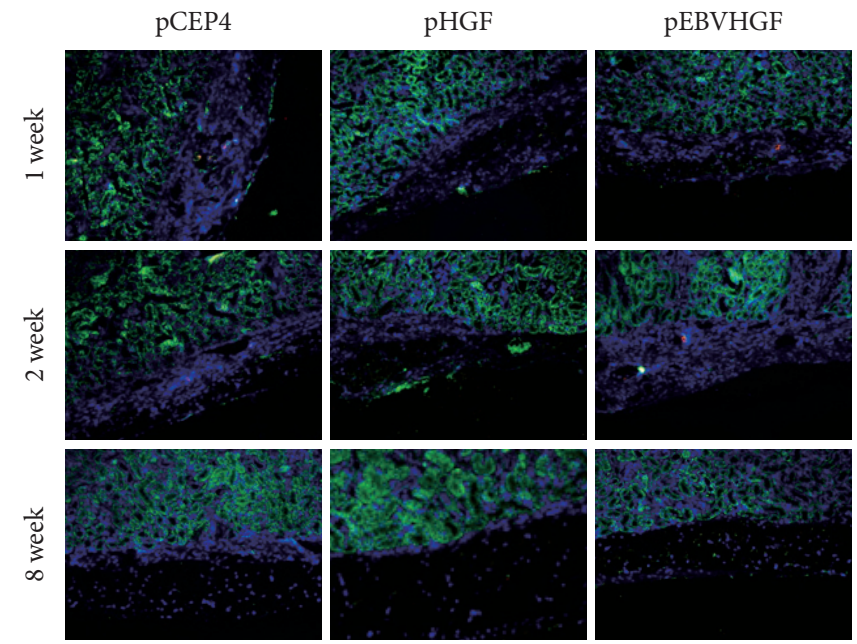

Fig. 7. Loss of pancreatic cells transplanted into normal mice. Pancreatic cells were transfected three times with pCEP4, pHGF, or pEBVHGF and then re-aggregated. Normal nude mice were transplanted with the re-aggregated pancreatic cells in the kidney capsule. The transplanted cells were examined using immunohistochemistry one, two, and eight weeks after transplantation. Insulin is shown in red and pancytokeratin is shown in green, while nuclear staining is shown in blue.

\section{DISCUSSION}

In this study, we observed that re-aggregated pancreatic cells showed improved engraftment than the cells in single-cell state when they were transplanted into immunodeficient mouse kidney capsules. These results are comparable to the results reported by Tatarkiewicz et al. [12]. Pancreatic cells in kidney capsules were unable to form islets two weeks after transplantation in our study, similar to what they [12] observed four weeks after transplantation. However, the cells appeared as dense cluster, and blood vessel formation within the cell cluster was observed at two weeks after transplantation. Also, some of the cells were able to produce insulin, although the majority was not. Glucose-stimulated insulin secretion was higher in pseudo-islet forms of MIN 6 cells, a type of $\beta$-cell, compared to what was observed in monolayer cultured MIN 6 cell [22]. Thus, re-aggregated pancreatic cells seem to be more effective for engraftment and insulin production possibly through the increased cell density and, interaction between cells.

When monolayer cultured and then re-aggregated pancreatic cells were transplanted, there was no islet formation after two and four weeks [12]. In contrast, the islets were formed when NPCCs were separated and immediately transplanted 
without further in vitro manipulation as shown in Fig. 1 and published by others [23-25]. These discrepancies might be due to the loss of extracellular matrix or other factors which are required for islet structure formation, during the NPCCs dispersion into single cells. Even if the cells are re-aggregated, these substances or factors might not be able to easily recovered [10, 12,26].

Unlike in the previous study where the re-aggregated pancreatic cells were simply transplanted into nude mouse [12], we transfected gene to monolayer cultured pancreatic cells and re-aggregated before transplantation to promote differentiation of duct cells into beta cells. Since the pancreatic cells are primary cells, to overcome the low transfection efficiency, we repeated the gene transfection. Even though repeated transfection resulted in decreased cell viability, the transfection efficiency was overall increased (data not shown). We expected transplanting pancreatic cells that were repeatedly transfected with genes, would facilitate beta cell differentiation and cell survival in vivo. However, transfected cells were unable to survive in diabetic mouse models, demonstrating that this approach is not a candidate for a possible diabetes treatment. During the first few days after transplantation of the cells transfected with pEBVHGF, slight drop in blood glucose was observed in diabetic nude mice. This is possible because the pancreatic cells remained intact at the early stage before experiencing fibrosis or apoptosis.

To test if high glucose toxicity hampered survival of pancreatic cells in the diabetes mouse models, we transplanted the transfected cells into normal nude mice. The pancreatic cells were unable to successfully engraft in this case, either. Thus, high glucose toxicity would not be the main cause of failed engraftment of pancreatic cells in vivo. There are many likely causes for the failure of engraftments. Damage to the cells caused by the increase in culture period, transfection, centrifugation, and other in vitro manipulation could be one reason. It took up to nine days till prepare cells for transplantation if they were monolayer cultured and re-aggregated without transfection. However, it took thirteen days if the pancreatic cells received three consecutive transfections, and then re-aggregated prior to transplantation. Therefore, a four-day increase in the in vitro incubation period, as well as exposure to the repeated lipofectamine transfection are thought to be the main interfering points in successful engraftment of re-aggregated cells. As the pancreatic cells that underwent transfection and re-aggregation expressed pancytokeratin and insulin, these processes seem to affect the cells further under in vivo conditions than during in vitro culture.

Our results demonstrate that when pancreatic cells repeatedly transfected with pEBVHGF were transplanted into diabetic animal models, no appreciable therapeutic effect was observed. In our in vitro experiments, when pancreatic cells were transfected with pEBVHGF, the HGF receptor was expressed and phosphorylated [20]. The increases in cell growth and in the expression of insulin were also confirmed [20]. Thus, further study is necessary to minimize the handing time from the separation of the pancreatic cells to transplantation into the animal models, and to reduce cell damage during gene transfection, re-aggregation, and the transplantation processes. Also, if we can develop a method for clustering the cells to form islet-like structures, successful results for diabetes treatments using neonatal porcine pancreatic cells may be achieved in the future.

\section{ACKNOWLEDGMENT}

This work was supported by a Korea Research Foundation grant funded by the Korean Government (KRF-2008-314C00254). The authors wish to acknowledge the financial support of the Catholic Institute of Cell Therapy Basic Science Programs Foundation made in the program year of 2007.

\section{REFERENCES}

1. Cavanagh TJ, Lakey JR, Wright MJ, Albertson T, Wile K, Fetterhoff TJ. Identification of a pig strain with maximal islet mass. Transplant Proc 1998;30:368.

2. Ohgawara H, Iwanaga T, Yui R, Nishijima S, Hirata Y. Monolayer-forming islet cell culture from neonatal pig pancreas: using sequential treatment with EDTA-dispase and monoiodoacetic acid for preparation and purification. Tohoku J Exp Med 1987;153:375-82.

3. Witzigmann H, Ludwig S, Armann B, Gabel G, Teupser D, Kratzsch J, Pietsch UC, Tannapfel A, Geissler F, Hauss J, Uhlmann D. Endothelin(A) receptor blockade reduces ischemia/ reperfusion injury in pig pancreas transplantation. Ann Surg 2003;238:264-74.

4. Omer A, Duvivier-Kali VF, Trivedi N, Wilmot K, Bonner-Weir S, Weir GC. Survival and maturation of microencapsulated porcine neonatal pancreatic cell clusters transplanted into immunocompetent diabetic mice. Diabetes 2003;52:69-75. 
5. Ohgawara H, Kobayashi A, Kawamura M, Karibe S, Fu Q, Omori Y, Akaike T. Development of a method for embeddedculture of pig pancreatic islet-like cell clusters in agarose containing maltose-carrying polystyrene (HEVM) and nicotinamide. Cell Transplant 1994;3:83-9.

6. Chen B, Liu J, Zhu X, Liu X. Transplantation of encapsulated neonatal porcine islet-like cluster cells into diabetic rats. Chin Med J (Engl) 1996;109:197-200.

7. Korsgren O, Jansson L, Sundler F. Reinnervation of transplanted fetal porcine endocrine pancreas. Evidence for initial growth and subsequent degeneration of nerve fibers in the islet grafts. Transplantation 1996;62:352-7.

8. Yoon KH, Quickel RR, Tatarkiewicz K, Weir GC, Bonner-Weir S. Growth and differentiation of transplanted porcine neonatal pancreatic cell clusters in normal nude mice. Transplant Proc 1998;30:601.

9. Weir GC, Quickel RR, Yoon KH, Tatarkiewicz K, Ulrich TR, Hollister-Lock J, Bonner-Weir S. Porcine neonatal pancreatic cell clusters (NPCCs): a potential source of tissue for islet transplantation. Ann Transplant 1997;2:63-8.

10. Korbutt GS, Elliott JF, Ao Z, Smith DK, Warnock GL, Rajotte RV. Large scale isolation, growth, and function of porcine neonatal islet cells. J Clin Invest 1996;97:2119-29.

11. Epidemiology of Diabetes Interventions and Complications (EDIC) Research Group. Effect of intensive diabetes treatment on carotid artery wall thickness in the epidemiology of diabetes interventions and complications. Diabetes 1999;48:383-90.

12. Tatarkiewicz K, Lopez-Avalos MD, Yoon KH, Trivedi N, Quickel RR, Bonner-Weir S, Weir GC. Development and retroviral transduction of porcine neonatal pancreatic islet cells in monolayer culture. Dev Growth Differ 2003;45:39-50.

13. Lopez-Avalos MD, Tatarkiewicz K, Sharma A, Bonner-Weir S, Weir GC. Enhanced maturation of porcine neonatal pancreatic cell clusters with growth factors fails to improve transplantation outcome. Transplantation 2001;71:1154-62.

14. Valdes-Gonzalez RA, Dorantes LM, Garibay GN, Bracho-Blanchet E, Mendez AJ, Davila-Perez R, Elliott RB, Teran L, White DJ. Xenotransplantation of porcine neonatal islets of Langerhans and Sertoli cells: a 4-year study. Eur J Endocrinol 2005; 153:419-27.

15. Rolo AP, Palmeira CM. Diabetes and mitochondrial function: role of hyperglycemia and oxidative stress. Toxicol Appl Pharmacol 2006;212:167-78.

16. Nakamura T, Nawa K, Ichihara A. Partial purification and char- acterization of hepatocyte growth factor from serum of hepatectomized rats. Biochem Biophys Res Commun 1984;122: 1450-9.

17. Nakamura T. Structure and function of hepatocyte growth factor. Prog Growth Factor Res 1991;3:67-85.

18. Boros P, Miller CM. Hepatocyte growth factor: a multifunctional cytokine. Lancet 1995;345:293-5.

19. Guido L, Basta G, Racanicchi L, Mancuso F, Luca G, Macchiarulo G, Brunetti P, Calafiore R. Short-term stimulation studies on neonatal pig pancreatic duct-derived cell monolayers. Transplant Proc 2005;37:2715-8.

20. Kim MS, Kim JW, Sun C, Oh ST, Yoon KH, Lee SK. Induction of efficient differentiation and survival of porcine neonatal pancreatic cell clusters using an EBV-based plasmid expressing HGF. J Biochem 2008;143:497-503.

21. Nanbo A, Sugden A, Sugden B. The coupling of synthesis and partitioning of EBV's plasmid replicon is revealed in live cells. EMBO J 2007;26:4252-62.

22. Hauge-Evans AC, Squires PE, Persaud SJ, Jones PM. Pancreatic beta-cell-to-beta-cell interactions are required for integrated responses to nutrient stimuli: enhanced $\mathrm{Ca} 2+$ and insulin secretory responses of MIN6 pseudoislets. Diabetes 1999;48: 1402-8.

23. Sun CL, Ham DS, Park HS, Kim JW, Cho JH, Song KH, Son HY, Yoon KH. Rapamycin suppresses the expansion and differentiation of porcine neonatal pancreas cell clusters. Transplantation 2010;90:717-24.

24. Trivedi N, Hollister-Lock J, Lopez-Avalos MD, O’Neil JJ, Keegan M, Bonner-Weir S, Weir GC. Increase in beta-cell mass in transplanted porcine neonatal pancreatic cell clusters is due to proliferation of beta-cells and differentiation of duct cells. Endocrinology 2001;142:2115-22.

25. Ko SH, Kwon HS, Suh SH, Yang JH, Kim SR, Ahn YB, Song KH, Yoo SJ, Son HS, Cha BY, Lee KW, Son HY, Kang SK, Park CG, Yoon KH. Dexamethasone suppresses the expansion and transdifferentiation of transplanted porcine neonatal pancreas cell clusters (NPCCs) into beta-cells in normal nude mice. Diabetes Res Clin Pract 2004;66 Suppl 1:S97-101.

26. Yoon KH, Quickel RR, Tatarkiewicz K, Ulrich TR, HollisterLock J, Trivedi N, Bonner-Weir S, Weir GC. Differentiation and expansion of beta cell mass in porcine neonatal pancreatic cell clusters transplanted into nude mice. Cell Transplant 1999; 8:673-89. 